\title{
Effects of anisotropy on the critical temperature in layered nonadiabatic superconductors
}

\author{
I.N. Askerzade a,b, B. Tanatar ${ }^{\mathrm{c}, *}$ \\ a Department of Physics, Ankara University, Tandogan, Ankara 06100, Turkey \\ ${ }^{\mathrm{b}}$ Institute of Physics, Azerbaijan National Academy of Sciences, H.Cavid-33, Baku 370143, Azerbaijan \\ ${ }^{\mathrm{c}}$ Department of Physics, Bilkent University, Bilkent, Ankara 06533, Turkey
}

Received 7 May 2002; accepted 8 July 2002

\begin{abstract}
The generalized anisotropic Eliashberg theory is employed to study the critical temperature of layered nonadiabatic superconductors where the relevant phonon energy is comparable to the Fermi energy. We consider a two-dimensional model appropriate for cuprate compounds and recently discovered superconductor magnesium-diboride $\left(\mathrm{MgB}_{2}\right)$ which also reveals layered structure. By using the McMillan approximation we present the result of calculations of critical temperature $T_{\mathrm{c}}$. It is shown that the critical temperature is enhanced due to the influence of anisotropy and nonadiabaticity.
\end{abstract}

(c) 2002 Elsevier Science B.V. All rights reserved.

Keywords: Nonadiabatic superconductors; Electron-phonon interactions; Layered systems; Migdal theorem

\section{Introduction}

In conventional superconductors validity of the Eliashberg equations [1] is determined by the parameter $m=\omega_{0} / E_{\mathrm{F}}$, the ratio of the relevant phonon energy $\omega_{0}$ to the Fermi energy $E_{\mathrm{F}}$. As shown by Migdal [2] as long as $m \ll 1$, the electron-phonon (e-ph) vertex corrections are at least of order $\lambda \omega_{0} / E_{\mathrm{F}}$, where $\lambda$ is the e-ph coupling constant, thus they can be neglected. The possible breakdown of the Migdal theorem in some unconventional superconductors makes it necessary

\footnotetext{
${ }^{*}$ Corresponding author. Tel.: +90-312-2901591; fax: +90312-2664579.

E-mail address: tanatar@fen.bilkent.edu.tr (B. Tanatar).
}

to generalize the Eliashberg theory beyond the $m \ll 1$ approximation. Taking these corrections into account has been termed nonadiabatic superconductivity. For high $T_{\mathrm{c}}$ superconductors of interest in the last decade or so, the experimental data [3] suggest that the Fermi energy ranges between $E_{\mathrm{F}} \sim 0.1-0.3 \mathrm{eV}$ and the Debye phonon energy is of the order of $\omega_{\mathrm{D}} \sim 0.08-0.16 \mathrm{eV}$, making the Migdal ratio $m$ not negligible. Although somewhat debated there is experimental evidence [4] that e-ph interactions construe the basic mechanism in high $T_{\mathrm{c}}$ superconductors. Existence of strong e-ph interaction in cuprate superconductors was confirmed by the recent observation of the subgap structure in tunnel Josephson junction experiments [5]. As discussed by Maximov et al. [6] similar phenomena occur 
due to the interaction of Josephson current with phonons. As shown by Maximov [7], the e-ph mechanism explains many features of low-energy relaxation process in the cuprate superconductors, including the high values of critical temperature. With these arguments in mind, a generalized Migdal-Eliashberg theory which includes the first nonadiabatic correction was introduced and developed in a series of papers by Pietronero and coworkers [8-10]. In a perturbative approach, they considered the variation of $T_{\mathrm{c}}$ with $m$ and e-ph coupling strength $\lambda$, for different values of the momentum transfer $q$. Their method has further been employed to investigate various other aspects of nonadiabaticity effects [11-13]. Attempts were also made to consider the e-ph vertex corrections within nonperturbative schemes [14-16]. Some contrasting results and predictions of these approaches are discussed recently by Cosenza et al. [16] and Danylenko and Dolgov [17].

In most of the previous works, the nonadiabatic superconductivity has been studied by requiring the order parameter to be independent of momenta. However, in unconventional superconductors there is a strong momentum dependence of the order parameter. For instance, the order parameter of several cuprate superconductors have a predominant d-wave symmetry $\Delta(\mathbf{k})=\Delta\left[\cos \left(k_{x} a\right)-\right.$ $\cos \left(k_{y} a\right)$ ]. It is well known [8-10] that the inclusion of nonadiabatic corrections to e-ph interaction leads to strong momentum dependence and this induced momentum dependence leads to an enhancement of the critical temperature $T_{\mathrm{c}}$. Influence of the d-wave symmetry on the above-mentioned behavior was studied by Paci et al. [18].

In this work we study how the anisotropy brought about by a layered structure and the pairing interaction affects the critical temperature. We use the generalized Migdal-Eliashberg theory as developed by Pietronero and coworkers [8-10] and consider the anisotropy of the layered systems relevant to the cuprate compounds. As mentioned by Ummarino and Gonnelli [11] violation of the Migdal theorem in cuprate compounds is moderate, thus the perturbative treatment of nonadiabaticity should be reasonable. We consider the momentum dependent generalization of the equations satisfied by the renormalization parameter, and using the McMillan approach [19] we calculate the transition temperature $T_{\mathrm{c}}$.

In the rest of the paper, we first outline the twodimensional model we consider and the formalism for generalized Eliashberg equations. We then present our results by calculating the superconducting transition temperature as a function of various parameters of interest.

\section{Model and theory}

Since the cuprate compounds of recent interest consist of layered structures we assume a dispersion relation appropriate for a layered system of the form [20]

$E(\mathbf{k})=\frac{\hbar^{2}\left(k_{x}^{2}+k_{y}^{2}\right)}{2 m^{*}}+2 t\left[1-\cos \left(k_{z} d\right)\right]$.

Here $m^{*}$ is the in-plane effective mass, $t$ is the transverse transfer matrix element from one layer to another (or tunneling integral), and $d$ is the lattice constant in the $z$-direction. Such an energy spectrum of carriers was used by Jiang and Carbotte [21] for the calculation of various properties in a layered superconductor. For $E>4 t$, the Fermi surface is open and the density of states $N(E)$ is constant. The phonon spectrum of the layered crystals is, generally speaking, anisotropic. The dispersion relation for longitudinal $\omega_{\mathrm{L}}\left(q, q_{z}\right)$ and transverse phonons $\omega_{\mathrm{T}}\left(q, q_{z}\right)$ are given by the following expressions

$\omega_{\mathrm{L}}^{2}\left(q, q_{z}\right)=u_{\|}^{2}\left(q_{x}^{2}+q_{y}^{2}\right)+2 \frac{u_{z}^{2}}{d}\left(1-\cos \left(q_{z} d\right)\right)$

and

$\omega_{\mathrm{T}}^{2}\left(q, q_{z}\right)=u_{z}^{2}\left(q_{x}^{2}+q_{y}^{2}\right)+2 \frac{u_{\mathrm{T}}^{2}}{d}\left[1-\cos \left(q_{z} d\right)\right]$,

in which the sound velocities satisfy the condition $u_{\|} \gg u_{\mathrm{T}}, u_{z}$. As mentioned elsewhere $[8,10]$ the functions appearing in the generalized Eliashberg equations are defined by an averaging procedure over the Fermi surface. In the case of energy spectrum of Eq. (1) this procedure is equivalent to integration

$\int_{0}^{\arcsin Q_{\mathrm{c}}} \mathrm{d} \phi \ldots=4 \int_{0}^{2 p_{0}^{*} Q_{\mathrm{c}}} \frac{\mathrm{d} q}{\left\{\left(2 p_{0}^{*}\right)^{2}-q^{2}\right\}^{1 / 2}} \ldots$ 
where $\left(p_{0}^{*}\right)^{2}=p_{0}^{2}-4 m^{*} t\left(l-\cos p_{z} d\right)$ and $\phi$ denotes the angle between $\mathbf{p}$ and $\mathbf{p}^{\prime}$ which is equal to $p_{0}^{*}$ (see Fig. 1 in Ref. [24]), and $Q_{\mathrm{c}}$ is the cut-off parameter for the phonon momentum transfer $Q_{\mathrm{c}}=q_{\mathrm{c}} / 2 k_{\mathrm{F}}$. It is clear that the region of phonon transfer momentum $q=2 p_{0}^{*}$ makes the major contribution to the integrals. With this last argument, the generalized Eliashberg equations for layered systems can be obtained $[8,10]$ using the Einstein spectrum of effective frequency $\omega_{0}$ which is determined by the following expression (we shall neglect the contribution of transverse acoustic phonons to the e-ph coupling, due to the fact that $u_{\|} \gg u_{\mathrm{T}}, u_{z}$ )

$$
\begin{aligned}
\omega_{0}= & \sqrt{\left\langle\omega^{2}\right\rangle_{\mathrm{FS}}} \\
= & \left\{\frac{\mathrm{d}}{2 \pi} \int_{-\pi / d}^{\pi / d} \mathrm{~d} q_{z} \frac{1}{Q_{\mathrm{c}}}\right. \\
& \left.\times \int_{0}^{Q_{\mathrm{c}}} \frac{\mathrm{d} Q}{\left\{1-Q^{2}\right\}^{1 / 2}} \omega_{\mathrm{L}}^{2}\left(2 p_{0}^{*} Q, q_{z}\right)\right\}^{1 / 2} \\
= & \left\{2 u_{\|}^{2}\left(p_{0}^{2}-4 m^{*} t\right)\left(\frac{\arcsin Q_{\mathrm{c}}}{Q_{\mathrm{c}}}-\sqrt{1-Q_{\mathrm{c}}^{2}}\right)\right. \\
& \left.+\frac{2 u_{z}^{2}}{\mathrm{~d}^{2}} \frac{\arcsin Q_{\mathrm{c}}}{Q_{\mathrm{c}}}\right\}^{1 / 2} .
\end{aligned}
$$

Now the generalized Eliashberg equations describing pairing in systems with cylindrical symmetry has the form

$$
\begin{aligned}
Z\left(p_{z}, \omega_{n}\right) \Delta\left(p_{z}, \omega_{n}\right) \\
=\pi T_{\mathrm{c}} \int_{-\pi}^{\pi} \frac{\mathrm{d}\left(p_{z}^{\prime} d\right)}{2 \pi} \sum_{m} \frac{\lambda_{\Delta}\left(p_{z}, p_{z}^{\prime}, \omega_{n}, \omega_{m}, Q_{\mathrm{c}} ; \omega_{0}, E\right)}{\left(\omega_{n}-\omega_{m}\right)^{2}+\omega_{0}^{2}} \\
\quad \times \omega_{0}^{2} \frac{\Delta\left(p_{z}^{\prime}, \omega_{m}\right)}{\left|\omega_{m}\right|} \frac{2}{\pi} \arctan \left[\frac{E}{2 Z\left(p_{z}^{\prime}, \omega_{m},\right)\left|\omega_{m}\right|}\right]
\end{aligned}
$$

and

$$
\begin{aligned}
Z\left(p_{z}, \omega_{n}\right)= & 1+\frac{\pi T_{\mathrm{c}}}{\omega_{n}} \int_{-\pi}^{\pi} \frac{\mathrm{d}\left(p_{z}^{\prime} d\right)}{2 \pi} \\
& \times \sum_{m} \frac{\lambda_{z}\left(p_{z}, p_{z}^{\prime}, \omega_{n}, \omega_{m}, Q_{\mathrm{c}} ; \omega_{0}, E\right)}{\left(\omega_{n}-\omega_{m}\right)^{2}+\omega_{0}^{2}} \\
& \times \omega_{0}^{2} \frac{\omega_{m}}{\left|\omega_{m}\right|} \frac{2}{\pi} \arctan \left[\frac{E}{2 Z\left(p_{z}^{\prime}, \omega_{m}\right)\left|\omega_{m}\right|}\right],
\end{aligned}
$$

in which $Z\left(p_{z}, \omega_{n}\right)$ is the renormalization parameter, $\Delta\left(p_{z}, \omega_{n}\right)$ is the energy gap, $E$ is the total bandwidth, so that the energy is defined in the interval $-E / 2<\varepsilon<E / 2$, and $\omega_{m}=(2 m-1) \pi k_{\mathrm{B}} T_{\mathrm{c}}$ with $m=0, \pm 1, \pm 2, \ldots$ are the Matsubara frequencies. Here we use the following usual notation for the effective couplings defined as

$$
\begin{aligned}
& \lambda_{\Delta}\left(p_{z}, p_{z}^{\prime}, \omega_{n}, \omega_{m}, Q_{\mathrm{c}} ; \omega_{0}, E\right) \\
& =\lambda\left(p_{z}, p_{z}^{\prime}\right)\left[1+2 \lambda\left(p_{z}, p_{z}^{\prime}\right) P_{\mathrm{v}}\left(p_{z}, p_{z}^{\prime}, \omega_{n}, \omega_{m}, Q_{\mathrm{c}} ; \omega_{0}, E\right)\right. \\
& \left.\quad+\lambda\left(p_{z}, p_{z}^{\prime}\right) P_{\mathrm{c}}\left(p_{z}, p_{z}^{\prime}, \omega_{n}, \omega_{m}, Q_{\mathrm{c}} ; \omega_{0}, E\right)\right]
\end{aligned}
$$

and

$$
\begin{aligned}
& \lambda_{z}\left(p_{z}, p_{z}^{\prime}, \omega_{n}, \omega_{m}, Q_{\mathrm{c}} ; \omega_{0}, E\right) \\
& \quad=\lambda\left(p_{z}, p_{z}^{\prime}\right)\left[1+\lambda\left(p_{z}, p_{z}^{\prime}\right) P_{\mathrm{v}}\left(p_{z}, p_{z}^{\prime}, \omega_{n}, \omega_{m}, Q_{\mathrm{c}} ; \omega_{0}, E\right)\right] .
\end{aligned}
$$

The expressions for the so-called vertex and cross functions, $P_{\mathrm{v}}$ and $P_{\mathrm{c}}$, respectively, in general case were given by Paci et al. [18]. The vertex and cross functions are expanded in terms of Fermi-surface harmonics [22], which form a complete, orthonormal set of functions at the Fermi surface. In the case of our model energy spectrum (Eq. (1)), Fermi-surface harmonics can be represented by $\cos \left(n p_{z} d\right)$. Anisotropic e-ph coupling parameter $\lambda\left(p_{z}, p_{z}^{\prime}\right)$ without the corrections in Eqs. (8) and (9) are expanded as

$$
\begin{aligned}
\lambda\left(p_{z}, p_{z}^{\prime}\right)= & \lambda_{00}+\lambda_{01} \cos \left(p_{z} d\right)+\lambda_{01} \cos \left(p_{z}^{\prime} d\right) \\
& +\lambda_{11} \cos \left(p_{z} d-p_{z}^{\prime} d\right),
\end{aligned}
$$

with $\lambda_{01}=\lambda_{10}$. As pointed out by one of us [23] and Nakhmedov [24] the nondiagonal elements of the e-ph interaction in layered systems with electron spectrum of Eq. (1), the quasi-twodimensional phonon spectra (Eqs. (2) and (3)) are proportional to $t / E_{\mathrm{F}}$. As shown in the previous works $[25,26]$, layered systems are characterized by the low frequency optical phonons, which correspond to the oscillations of planes as rigid molecules with respect to each other. It was pointed by Bergman and Rainer [27], Dubovskii and Kozlov [28], and Alien and Dynes [22] that low frequency phonons play a significant role in superconductors with weak e-ph coupling. In the opposite case, i.e. in the strong 
coupling limit, the critical temperature $T_{\mathrm{c}}$ is determined by the high frequency peculiarities in the phonon spectrum. With these arguments in mind, we take into account the interaction of electrons with longitudinal acoustical in-plane phonons (Eq. (2)).

For layered systems, the above condition implies that $\lambda_{11} \ll \lambda_{01}<\lambda_{00}$, which suggests that in subsequent calculations we can neglect terms of order $\lambda_{11} / \lambda_{01}$ and $\lambda_{11} / \lambda_{00}$. For the calculation of $\lambda_{00}$ and $\lambda_{01}$ we will use the expression for e-ph interaction without the vertex correction in Eq. (10). In a more general situation we have the following expressions for the vertex corrected interaction (for convenience in Eqs. (8) and (9) other arguments are suppressed)

$$
\begin{aligned}
\lambda_{\Delta}\left(p_{z}, p_{z}^{\prime}\right)= & \lambda\left(p_{z}, p_{z}^{\prime}\right)\left[1+2 \sum_{k_{z}} \lambda\left(k_{z}-p_{z}\right) G\left(k_{z}\right)\right. \\
& \left.\times G\left(p_{z}^{\prime}-p_{z}+k_{z}\right)\right]+\lambda\left(p_{z}, p_{z}^{\prime}\right) \\
& \times \sum_{k_{z}} \lambda\left(k_{z}-p_{z}\right) G\left(k_{z}\right) G\left(k_{z}-p_{z}-p_{z}^{\prime}\right)
\end{aligned}
$$

and

$$
\begin{array}{r}
\lambda_{z}\left(p_{z}, p_{z}^{\prime}\right)=\lambda\left(p_{z}, p_{z}^{\prime}\right)\left[1+\sum_{k_{z}} \lambda\left(k_{z}-p_{z}\right)\right. \\
\left.\times G\left(k_{z}\right) G\left(k_{z}-p_{z}+p_{z}^{\prime}\right)\right] .
\end{array}
$$

For the small parameter $t / T_{\mathrm{c}} \ll 1$, and at temperatures close to $T_{\mathrm{c}}$, the Green's functions of electrons can be expressed as

$$
\begin{aligned}
G\left(\mathrm{i} \omega_{n}, p, p_{z}\right) & =\frac{1}{\mathrm{i} \omega_{n}-\xi\left(p, p_{z}\right)} \\
& \approx \frac{1}{\mathrm{i} \omega_{n}-\xi(p)}\left[1+\frac{t \cos \left(p_{z} d\right)}{\mathrm{i} \omega_{n}-\xi(p)}\right],
\end{aligned}
$$

where $\xi\left(p, p_{z}\right)-E\left(p, p_{z}\right)-\mu$, and $\mu$ being the chemical potential. Taking into account the expression given in Eqs. (10)-(12), we obtain the final expression for the vertex corrected e-ph interaction

$$
\begin{aligned}
\lambda_{\Delta}= & \lambda_{00}+\lambda_{00}^{2}\left(2 P_{\mathrm{v}}-P_{\mathrm{c}}\right) \\
& +\lambda_{01}\left(1-2 \lambda_{00}\left(2 P_{\mathrm{v}}+P_{\mathrm{c}}\right)\right) \cos \left(p_{z} d\right) \\
& +\lambda_{10}\left(1+\lambda_{00}\left(2 P_{\mathrm{v}}+P_{\mathrm{c}}\right)\right) \cos \left(p_{z}^{\prime} d\right) \\
& +\lambda_{00} \lambda_{10}\left(2 P_{\mathrm{v}}+P_{\mathrm{c}}\right) \cos \left(p_{z} d-p_{z}^{\prime} d\right)
\end{aligned}
$$

and

$\lambda_{z}=\lambda_{00}+\lambda_{00}^{2} P_{\mathrm{v}}$.

Within the model of Fermi-surface harmonics, the order parameter takes the form

$\Delta\left(p_{z}, \omega\right)=\Delta(\omega)+\Delta_{1}(\omega) \cos \left(p_{z} d\right)$.

As shown by Grimaldi et al. [8] the critical temperature $T_{\mathrm{c}}$ can be obtained from the generalized Eliashberg equations by an analytical approach. The final expression for $T_{\mathrm{c}}$ beyond the adiabatic limit in s-wave isotropic superconductors for arbitrary momentum transfer is given by [8]

$$
\begin{aligned}
T_{\mathrm{c}}= & \frac{1.13 \omega_{0}}{(1+m) \mathrm{e}^{1 / 2}} \exp \left[\frac{m}{(2+2 m)}\right] \\
& \times \exp \left[-\frac{1+\lambda_{z} /(1+m)}{\lambda_{\Delta}}\right] .
\end{aligned}
$$

Substituting Eqs. (14)-(16) into Eqs. (6) and (7), and making use of the McMillan approximation [19] we have a system of algebraic equations

$$
\left(1+\lambda_{z}^{00} /(1+m)-\lambda_{\Delta}^{11} x\right) \Delta_{0}+\lambda_{\Delta}^{10} x \Delta_{1}=0
$$

and

$\lambda_{\Delta}^{10} x \Delta_{0}+\left(1+\lambda_{z}^{00} /(1+m)-\lambda_{\Delta}^{11} x\right) \Delta_{1}=0$,

where

$x=\frac{\ln (1.13) \omega_{0}}{T_{\mathrm{c}}}-\ln (1+m)-\frac{\left(1-\frac{m}{1+m}\right)}{2}$

and

$\lambda_{z}^{00}=\lambda_{00}+\lambda_{00}^{2} P_{\mathrm{v}}$,

$\lambda_{\Delta}^{00}=\lambda_{00}+\lambda_{00}^{2}\left(2 P_{\mathrm{v}}+P_{\mathrm{c}}\right)$,

$\lambda_{\Delta}^{01}=\lambda_{01}+2 \lambda_{00} \lambda_{01}\left(2 P_{\mathrm{v}}+P_{\mathrm{c}}\right)$,

$\lambda_{\Delta}^{11}=\lambda_{00} \lambda_{11}\left(2 P_{\mathrm{v}}+P_{\mathrm{c}}\right)$.

From the condition of vanishing of the determinant of system of equations (Eqs. (18) and (19)) 
and the condition $t / E_{\mathrm{F}} \ll 1$, we obtain the following explicit formula for the critical temperature

$\frac{T_{\mathrm{c}}}{T_{\mathrm{c} 0}}=\exp \left(\kappa\left(\lambda_{01} / \lambda_{00}\right)^{2}\right)$,

where $T_{\mathrm{c} 0}$ is the critical temperature without the vertex corrections and

$$
\begin{aligned}
\kappa= & \frac{1}{2}\left(\frac{1+\lambda_{00}\left(1+\lambda_{00} P_{\mathrm{v}}\right) /(1+m)}{\lambda_{00}\left(1+\lambda_{00}\left(2 P_{\mathrm{v}}+P_{\mathrm{c}}\right)\right)}\right) \\
& \times\left(\frac{\left(1+\lambda_{00} P_{\mathrm{v}}\right) /(1+m)}{\lambda_{00}\left(1+\lambda_{00}\left(2 P_{\mathrm{v}}+P_{\mathrm{c}}\right)\right)}\right) .
\end{aligned}
$$

The coefficient $\kappa$ embodies the effects of vertex corrections and anisotropy in determining $T_{\mathrm{c}}$. The explicit forms of the vertex correction $P_{\mathrm{v}}$ and cross correction $P_{\mathrm{c}}$ for the two-dimensional case are presented by Paci et al. [18].

\section{Results and discussion}

Our main result for the effects of anisotropy on the critical temperature in layered nonadiabatic superconductors is given by Eq. (25). To assess these effects more quantitatively, we show in Fig. $1, T_{\mathrm{c}} / T_{\mathrm{c} 0}$ as a function of $\lambda_{01} / \lambda_{00}$, for different values of $Q_{\mathrm{c}}$ (where $Q_{\mathrm{c}}$ is the cut-off parameter of phonon momentum transfer). The explicit expres-

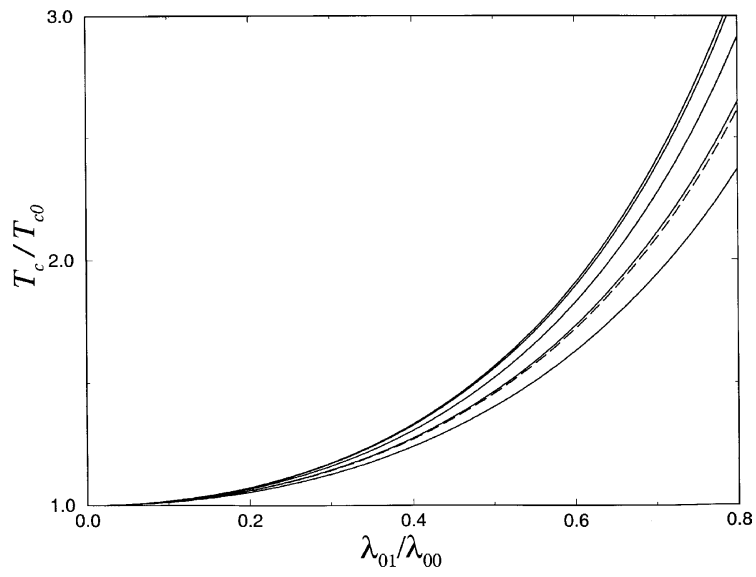

Fig. 1. The behavior of $T_{\mathrm{c}} / T_{\mathrm{c} 0}$ as a function of $\lambda_{01} / \lambda_{00}$, for $\lambda_{00}=0.5$ and $m=0.2$. The dashed line is for the case without vertex corrections. The solid lines are for different values of the cut-off parameter $Q_{\mathrm{c}}=0.9,0.7,0.5,0.3$, and 0.1 , from bottom to top. sions for $\lambda_{00}$ and $\lambda_{01}$ with the energy spectrum of Eq. (1) were presented in Ref. [23]. These expressions embody microscopic parameters which may be obtained from the experimental data (for example, $\left.u_{\|}, u_{z}, E_{\mathrm{F}}\right)$. However, our final expression for the critical temperature $T_{\mathrm{c}}$ given in Eq. (25) contains only the ratio of the parameters $\lambda_{01} / \lambda_{00}$. For the case of two-dimensional superconductors we take $\lambda_{00}=0.5$. In this figure the dashed curve denotes the behavior of $T_{\mathrm{c}}$ without the vertex corrections. Solid curves are for different $Q_{\mathrm{c}}$ values in the range $0.1-0.9$, from top to bottom, respectively. We observe that the nonadiabatic corrections become more prominent for small values of $Q_{\mathrm{c}}$. Note that $\kappa$ increases as $Q_{\mathrm{c}}$ decreases. For the value $Q_{\mathrm{c}}=0.9$, the coefficient $\kappa$ becomes lower than that in the adiabatic case. Thus, the vertex corrections have similar behavior in the anisotropic and isotropic superconductors when $Q_{\mathrm{c}}$ is small. The critical temperature in the nonadiabatic case is enhanced compared to the solution without the vertex and cross corrections.

Here we will discuss briefly the limits of using Fermi averaged acoustic phonons in the nonadiabatic limit. According to Madhukar [29], the magnitude of the vertex correction $P_{\mathrm{v}}$ in two dimensions for small phonon momentum transfer is

$P_{\mathrm{v}} \simeq \lambda\left\langle\frac{\omega^{1 / 2}(q)}{v_{\mathrm{F}}^{1 / 2} q^{1 / 2}}\right\rangle$.

Effective phonon frequency $\omega_{0}$ at small $Q_{\mathrm{c}}$ and $u_{\|} \gg u_{z}$ is given as $\omega_{0} \simeq u_{\|}\left(p_{0}^{2}-4 m^{*} t\right)^{1 / 2} Q_{\mathrm{c}}$. The averaging procedure over the Fermi surface for the quantity $\left\langle\frac{1}{q^{1 / 2}}\right\rangle$ is given by the following expression

$$
\begin{aligned}
\left\langle\frac{1}{Q^{1 / 2}}\right\rangle & =\frac{1}{Q_{\mathrm{c}}} \int_{0}^{Q_{\mathrm{c}}} \frac{\mathrm{d} Q}{\left\{1-Q^{2}\right\}^{1 / 2}} \frac{1}{Q^{1 / 2}} \\
& \simeq \frac{1}{Q_{\mathrm{c}}^{1 / 2}} .
\end{aligned}
$$

As follows from Eqs. (27) and (28) the classical Migdal result with effective phonon frequency remains unchanged. Thus, nonadiabatic effects for acoustic phonons become important for small values of phonon momentum transfer. However, as shown by Karakozov and Maksimov [30] strong nonadiabatic effects are possible for high phonon 


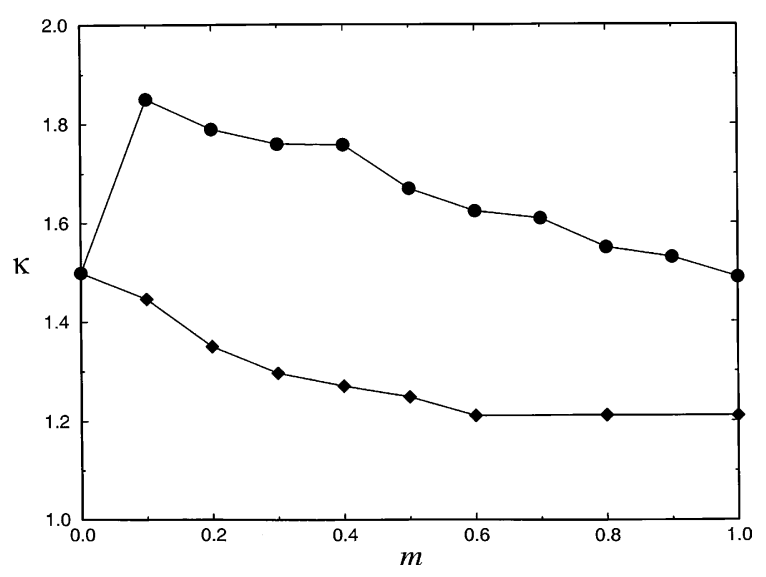

Fig. 2. The behavior of the coefficient $\kappa$ as a function of the nonadiabaticity parameter $m$ for different values of the momentum transfer $Q_{\mathrm{c}}=0.9$ (solid squares) and $Q_{\mathrm{c}}=0.1$ (solid circles). Lines joining the symbols are drawn to guide the eye.

momentum transfer with wave vector $\mathbf{q}$, which coincide with nesting wave vector $\mathbf{Q}_{\text {nest }}$. Energy spectrum of Eq. (1) exhibits nesting properties, but here nonadiabaticity related to nesting is not considered.

Dependence of $\kappa$ on the nonadiabaticity parameter $m$ is displayed in Fig. 2 for two different values of $Q_{\mathrm{c}}=0.1$ and 0.9 . As can be seen in the figure, in both cases $\kappa$ decreases with increasing $m$. At small $m$, corrections become more significant and they are reduced by increasing $m$. The behavior of $\kappa$ for other values of $Q_{\mathrm{c}}$ is similar to that shown in Fig. 2. These results we obtain seem interesting and relevant in connection with cuprate compounds as layered nonadiabatic superconductors. Another popular layered superconductor is $\mathrm{Sr}_{2} \mathrm{RuO}_{4}$ with $T_{\mathrm{c}} \approx 1 \mathrm{~K}$, which is rather low [31]. The layered structure of this system leads to a nearly cylindrical Fermi surface which is open along the $c$-axis. However, there are various indications for strong correlations and nonadiabaticity effects are absent in $\mathrm{Sr}_{2} \mathrm{RuO}_{4}$ compounds. Thus, in isotropic single-band s-wave nonadiabatic superconductors vertex corrections are strongly dependent on the momentum transfer and small values of $Q_{\mathrm{c}}$ lead to an enhancement of the critical temperature $T_{\mathrm{c}}$ [8-10].

As a concluding remark, it is interesting to add some considerations on the newly discovered su- perconductor magnesium diboride [32]. This material also has a layered structure with the boron atoms forming layers of two-dimensional honeycomb lattices. However, the Fermi level $(0.5 \mathrm{eV})$ crosses the in-plane $\sigma$ bands leading to a quasitwo-dimensional character of the electronic properties [33]. High phonon frequency of the boron atoms $\left(\omega_{\mathrm{ph}}=0.1 \mathrm{eV}\right)$ indicates that $\mathrm{MgB}_{2}$ could be in the nonadiabatic regime of the e-ph interaction [34]. An additional interesting point is that the superconductivity in $\mathrm{MgB}_{2}$ has two band and anisotropic character. Two band conventional Eliashberg theory for the $\mathrm{MgB}_{2}$ was supposed by Shulga et al. [35] for the study of upper critical field problem in $\mathrm{MgB}_{2}$. Investigations of nonadiabaticity in the framework of generalized isotropic Eliashberg equations was conducted [36] which are applicable for bulk samples of $\mathrm{MgB}_{2}$. Recent studies with the growth of single crystals [37] show anisotropy of physical properties in $\mathrm{MgB}_{2}$. From this point view our calculations seem attractive for the future study of nonadiabaticity effects in layered $\mathrm{MgB}_{2}$.

In this paper we have studied the problem of the momentum dependence of the nonadiabatic corrections for the a cylindrical symmetry of the order parameter. It is shown that in this case, inclusion of the nonadiabatic corrections enhances $T_{\mathrm{c}}$ compared to that without the vertex correction. There are various approximations in our presentation which may be improved in future calculations. Exact numerical solution [38] of the equations satisfied by the renormalization function $Z\left(p_{z}, \omega_{n}\right)$ should yield more quantitative results for the critical temperature $T_{\mathrm{c}}$. We believe, however, the essential behavior of $T_{\mathrm{c}}$ on the nonadiabaticity parameter $m$ for anisotropic superconductors should remain qualitatively the same. Finally, it should be possible to extend the ideas and formalism employed in this work to study the nonadiabatic corrections in other types of electronboson mechanisms.

\section{Acknowledgements}

This work was partially supported by the Scientific and Technical Research Council of Turkey 
(TUBITAK) under grant no. TBAG-2005, by NATO under grant no. SfP971970, and by the Turkish Department of Defense under grant no. KOBRA-001. I.N.A. acknowledges the support of TUBITAK NATO-PC grant and thanks the hospitality of Bilkent University. We thank Dr. T. Senger for useful discussions.

\section{References}

[1] G.M. Eliashberg, Sov. Phys. JETP 11 (1960) 696.

[2] A.B. Migdal, Sov. Phys. JETP 7 (1958) 996.

[3] Y.J. Uemura et al., Phys. Rev. Lett. 66 (1991) 2665; N. D'Ambrumenil, Nature (London) 352 (1991) 472.

[4] J.P. Franck, S. Harker, J.H. Brewer, Phys. Rev. Lett. 71 (1993) 283 ;

M.K. Craford et al., Science 250 (1990) 1390.

[5] Ya.G. Ponomarev et al., Solid State Commun. 111 (1999) 513.

[6] E.G. Maximov, P.I. Areseyev, N.S. Malova, Solid State Commun. 111 (1999) 391.

[7] E.G. Maximov, Usphekhi Fiz. Nauk 170 (2000) 1033.

[8] C. Grimaldi, L. Pietronero, S. Strässler, Phys. Rev. Lett. 75 (1995) 1158.

[9] L. Pietronero, S. Strässler, C. Grimaldi, Phys. Rev. B 52 (1995) 10516.

[10] C. Grimaldi, L. Pietronero, S. Strässler, Phys. Rev. B 52 (1995) 10530.

[11] G.A. Ummarino, R.S. Gonnelli, Physica C 317-318 (1999) 524 ;

Int. J. Mod. Phys. B 13 (1999) 1215;

Phys. Rev. B 56 (1997) R14279.

[12] S. Sarkar, Phys. Rev. B 57 (1998) 11661.

[13] M.E. Palistrant, F.G. Kochorbe, J. Phys.: Condens. Matter 12 (2000) 2217.

[14] J. Cai, X.L. Lei, L.M. Xie, Phys. Rev. B 39 (1989) 11618.

[15] Y. Takada, J. Phys. Chem. Solids 54 (1993) 1779; Phys. Rev. B 52 (1995) 12708.
[16] F. Cosenza, L. De Cesare, M. Fusco Girard, Phys. Rev. B 59 (1999) 3349; Phys. Lett. A 266 (2000) 83.

[17] O.V. Danylenko, O.V. Dolgov, Phys. Rev. B 63 (2001) 094506.

[18] P. Paci, C. Grimaldi, L. Pietronero, Eur. Phys. J. B 17 (2000) 235.

[19] W.L. McMillan, Phys. Rev. 167 (1968) 331.

[20] E.P. Nakhmedov, O.A. Sacli, I.N. Askerzade, J. Phys.: Condens. Matter 5 (1993) 1099.

[21] J. Jiang, J.P. Carbotte, Phys. Rev. B 53 (1996) 12400; Phys. Rev. B 48 (1993) 3853;

Phys. Rev. B 45 (1992) 10670; Physica C 210 (1993) 325.

[22] P.B. Allen, R.C. Dynes, Phys. Rev. B 12 (1975) 905.

[23] I.N. Askerzade, Proceedings of Nathiagali Summer College, Pakistan, June 1996, p. 11.

[24] E.P. Nakhmedov, Phys. Rev. B 54 (1996) 6624.

[25] L.D. Landau, E.M. Lifshitz, Statistical Physics, vol. 5, Pergamon, New York, 1980.

[26] G.L. Belenkii, E.Yu. Salaev, R.A. Suleymanov, Usphekhi Fiz. Nauk 155 (1988) 89.

[27] G. Bergman, D. Rainer, Z. Phys. 263 (1973) 59.

[28] L.B. Dubovski, A.N. Kozlov, Zh. Eksp. Theor. Fiz. 68 (1975) 2224

[29] A. Madhukar, Solid State Commun. 24 (1977) 11.

[30] A.E. Karakozov, E.G. Maksimov, JETP 88 (1999) 987.

[31] A.P. Mackenzie, J. Supercond. 12 (1999) 543.

[32] J. Nagamatsu, N. Nakagawa, T. Muranaka, Y. Zenitani, J. Akimitsu, Nature (London) 410 (2001) 63.

[33] J.M. An, W.E. Pickett, Phys. Rev. Lett. 86 (2001) 4366.

[34] A.S. Alexandrov, Physica C 363 (2001) 231.

[35] S.V. Shulga, S.-L. Drechsler, H. Eschrig, H. Rosner, W.E. Pickett, cond-mat/0103154.

[36] E. Cappelluti, S. Ciuchi, C. Grimaldi, L. Pietronero, S. Strässler, Phys. Rev. Lett. 88 (2002) 117003.

[37] M. Zehermayer, M. Elstever, H.W. Weber, J. Jun, S.M. Kazakov, J. Karpinski, A. Wisniewski, cond-mat/0204199.

[38] See for instance V.N. Kostur, B. Mitrovic, Phys. Rev. B 50 (1994) 12774;

O.V. Danylenko, O.V. Dolgov, M.L. Kulic, V. Oudovenko, Eur. Phys. J. B 9 (1999) 201. 